FORMATLON Formation emploi

Revue française de sciences sociales

124 | Octobre-Décembre 2013

Pêle-mêle

\title{
Les suppressions d'emplois dans la filière automobile : l'impact négatif sur les conditions de travail et la qualification ouvrière
}

Les suppressions d'emplois dans la filière automobile : l'impact négatif sur les conditions de travail et la qualification ouvrière Loss of jobs in the automotive industry: negative impact on working conditions and workers' qualifications

Arbeitsplatzabbau in der Automobilindustrie: negative Auswirkungen auf Arbeitsbedingungen und Qualifizierung der Arbeiter

Las supresiones de empleos en el sector automotriz : el impacto negativo sobre las condiciones de trabajo y la calificación obrera

\section{Armelle Gorgeu et René Mathieu}

\section{(2) OpenEdition}

Journals

Édition électronique

URL : http://journals.openedition.org/formationemploi/4093

DOI : 10.4000/formationemploi.4093

ISSN : 2107-0946

Éditeur

La Documentation française

Édition imprimée

Date de publication : 31 décembre 2013

Pagination : 87-103

ISSN : 0759-6340

Référence électronique

Armelle Gorgeu et René Mathieu, «Les suppressions d'emplois dans la filière automobile : l'impact négatif sur les conditions de travail et la qualification ouvrière », Formation emploi [En ligne], 124 | Octobre-Décembre 2013, mis en ligne le 08 janvier 2015, consulté le 30 octobre 2020. URL : http:// journals.openedition.org/formationemploi/4093; DOI : https://doi.org/10.4000/formationemploi.4093 


\title{
Les suppressions d'emplois dans la filière automobile : l'impact négatif sur les conditions de travail et la qualification ouvrière
}

\author{
Armelle Gorgeu \\ Chercheur au Centre de recherches sociologiques et politiques de Paris (CRESPPA), laboratoire \\ rattaché au CNRS (UMR 7217), et à l'université Paris VIII. Elle est socio-économiste \\ René Mathieu \\ Chercheur au Centre de recherches sociologiques et politiques de Paris (CRESPPA), laboratoire \\ rattaché au CNRS (UMR 7217), et à l'université Paris VIII. Il est socio-économiste
}

Résumé

Les suppressions d'emplois dans la filière automobile : l'impact négatif sur les conditions de travail et la qualification ouvrière

Cet article, basé sur des entretiens récents, met en relief une relation étroite entre les suppressions d'emplois dans les usines de la filière automobile, la détérioration des conditions de travail et la remise en cause de la qualification ouvrière, et ce, en raison d'une standardisation accrue du mode opératoire et d'une intensification du travail. La majorité des ouvriers ayant fait l'objet d'entretiens depuis 2006 font état de conditions de travail dégradées, d'une faible reconnaissance de leurs compétences, et certains se sentent déclassés.

Mots clés : suppression d'emplois, industrie automobile, conditions de travail, qualification, ouvrier

Abstract

Loss of jobs in the automotive industry: negative impact on working conditions and workers' qualifications

This article, based on recent interviews, highlights a close relationship between the loss of jobs in car manufacturing plants, deterioration of working conditions and calling into question of workers' qualifications, due to an increased standardisation of procedures and an intensification of work. The majority of workers interviewed since 2006 reports deteriorated working conditions, poor acknowledgement of their skills and some even feel downgraded. 
Keywords: job cuts, automotive industry, working condition, qualification, worker Journal of Economic Literature: M 51

Traduction : TSF

Cet article s'interroge sur les effets des suppressions d'emplois sur les conditions de travail et la qualification des ouvriers en prenant l'exemple de la filière automobile, c'està-dire des usines des constructeurs et de leurs fournisseurs, sur la période 2006-2011, où la dégradation de l'emploi a été particulièrement importante. Dans cette filière, les transformations ont été continues depuis les années 90 ; elles peuvent être qualifiées de restructurations lorsqu'elles affectent à la fois l'emploi et l'organisation du travail et se sont intensifiées depuis une dizaine d'années. Cette intensification est due à de nombreuses délocalisations d'activités dans les pays à bas salaires, qui ont provoqué, chez les fournisseurs, des fermetures d'usines et d'ateliers, et une réduction importante de l'effectif ouvrier au cours de la seconde moitié des années 2000, notamment depuis la crise financière de 2008. L'évolution de l'emploi dans la filière automobile est à replacer dans le cadre des transformations de la structure de l'emploi en France depuis une trentaine d'années, et notamment de 2000 à 2008 où la part de l'industrie dans l'emploi total passe de $16 \%$ à $13 \%{ }^{1}$.

Cet article s'appuie sur des recherches récentes ( $c f$. encadré 1) qui prolongent des travaux qualitatifs menés depuis plus de vingt ans sur l'organisation du travail et la gestion de l'emploi et de la main-d'œuvre dans les usines de la filière automobile. Ces derniers faisaient état d'une déqualification ouvrière qui procédait de plusieurs facteurs : réorganisations, renouvellement des directions, recours à l'intérim, exigences de polyvalence et de polycompétence, sélectivité à l'embauche et segmentation entre les emplois. Pour les responsables dans les usines, il n'y avait pas d'évolution linéaire en matière de qualification ouvrière en raison des réorganisations incessantes ; en outre, l'automatisation pouvait être évoquée par certains comme une cause de déqualification en raison de l'augmentation de la fiabilité des machines. Pour les responsables syndicaux, la remise en cause de la qualification ouvrière était due surtout à la suppression d'emplois ouvriers hautement qualifiés et à l'émergence, en contrepartie, d'emplois aux contours flous n'ayant pas fait l'objet de négociations collectives, mais aussi à l'uti-

1. Depuis 1978 , l'emploi industriel a connu une baisse quasiment ininterrompue de $1,3 \%$ par an en moyenne, soit une destruction annuelle de 60000 postes quand les services marchands en créaient 150000 (Bouvier, Pilarski, 2008). La direction du Trésor, dans le rapport sur la désindustrialisation en France (Demmou, 2010) estime que deux millions d'emplois industriels ont disparu en trente ans. Sur la période 20002008, l'emploi dans l'industrie a diminué de 500000 personnes, c'est-à-dire de $13 \%$, passant de 3,86 millions de salariés à 3,36 millions (Gauron, 2010). La crise financière de 2008 a accéléré la détérioration de l'emploi, 269000 emplois ayant été supprimés dans l'industrie entre début 2008 et fin 2009 (Duval, 2010), soit une baisse de $7,7 \%$. 
lisation massive d'intérimaires ; en effet, un poste considéré comme qualifié devient déqualifié quand un intérimaire l'occupe.

\section{Encadré 1}

\section{Sources d'information}

Nous mobilisons les résultats de deux recherches qualitatives centrées sur les conditions de travail des ouvriers, effectuées dans le cadre des post enquêtes SUMER (Surveillance médicale des expositions aux risques professionnels) 2003 et 2010 ; cela permettait d'interroger des ouvriers ayant fait l'objet de cette enquête (réalisée par des médecins du travail au cours d'une visite médicale) et ayant accepté de recevoir des chercheurs à leur domicile.

La sélection était faite en fonction du secteur d'activité, du genre, et du statut (CDI - contrat à durée indéterminée, et intérimaires). Les entretiens auprès des ouvriers, réalisés par les responsables de la recherche et enregistrés au magnétophone, étaient complétés par d'autres auprès de médecins du travail, de représentants syndicaux, et de responsables des usines où travaillaient ces ouvriers dans le cas de la première recherche.

L'objectif de la première recherche, effectuée en 2006-2007 à partir de l'enquête SUMER 2003 dans la filière automobile et dans les industries agroalimentaires (Ardenti, Mathieu, Gorgeu 2010), était d'étudier les conditions de travail des ouvriers et leurs effets sur la santé en centrant l'analyse sur les relations entre donneurs d'ordres et fournisseurs et sur les trajectoires des ouvriers interrogés.

Dans le cas de la filière automobile, cette recherche a été menée dans les régions Bretagne, Franche Comté, Haute et Basse Normandie, Lorraine, et Nord-Pas-de-Calais. Elle s'appuie sur des entretiens auprès de trente et un ouvriers (dont dix femmes et huit intérimaires), travaillant dans des usines constructeurs (neuf personnes en $\mathrm{CDI}$ ) et dans des usines fournisseurs (quatorze personnes en $\mathrm{CDI}$ ), la plupart des intérimaires ayant effectué des missions dans ces deux types d'usines ; auprès de responsables de huit usines (une usine d'un constructeur et sept usines de fournisseurs); auprès de cinq responsables syndicaux et de cinq médecins du travail.

La seconde recherche, à partir de l'enquête SUMER 2010, est basée sur des entretiens réalisés d'octobre 2010 à avril 2011 dans les régions Bretagne, Franche Comté, Lorraine et Nord-Pas de-Calais. Elle avait pour objectif d'étudier les effets de la récession de 2009 sur les conditions de travail des ouvriers de la filière automobile (Mathieu, Gorgeu, 2012). Les suppressions d'emplois et leurs répercussions en termes d'organisation du travail, de changements de postes, de rythme de travail, de charge mentale, de marge d'initiative, de compétences et de santé étaient les principaux thèmes du guide d'entretien. Trente-quatre entretiens ont été effectués, dix-neuf avec des ouvriers (dont sept femmes), sept avec des médecins du travail interentreprises, et huit avec des représentants syndicaux. Aucun intérimaire n'a pu être interrogé car ceux que nous avons pu contacter ont refusé l'entretien.

Ces travaux datent d'avant la forte détérioration de l'emploi qui a marqué cette filière depuis $2006^{2}$; ainsi, il semble pertinent aujourd'hui d'élargir la réflexion sur le ressenti de

2. La filière automobile connaît, depuis 2006, une crise structurelle due à la mondialisation des marchés et des capitaux qui a été aggravée par la récession de 2009. Les suppressions d'emplois ont été nombreuses 
l'impact des suppressions d'emplois sur la qualification ouvrière à partir d'entretiens plus récents auprès d'ouvriers et ouvrières mais aussi de responsables des ressources humaines, de représentants syndicaux, et de médecins du travail. Ces travaux se centraient sur les conditions de travail, même si le thème de la qualification était sous-jacent. Les recherches menées depuis 2006 mettent en relief une relation étroite entre les suppressions d'emplois, la détérioration des conditions de travail et la remise en cause de la qualification ouvrière. Les résultats de ces travaux qualitatifs sur la filière automobile sont à rapprocher d'autres, notamment statistiques, dont le champ est beaucoup plus large. Ainsi les enquêtes européennes les plus récentes sur les conditions de travail montrent que la qualité de vie au travail s'est dégradée entre 1995 et 2005 (Greenam, Kalugina, Walkowiak, 2011). Les pénibilités physiques ont augmenté, l'intensité des contraintes s'est accrue, et les tâches sont devenues moins enrichissantes pour les travailleurs.

La première partie de cet article met en relief l'impact des suppressions d'emplois sur le contenu du travail des ouvriers, à savoir une standardisation du mode opératoire perçue comme une déqualification, une intensification du travail, et une plus grande responsabilisation sans compensation. La seconde partie montre que plusieurs ouvriers et ouvrières se sentent déclassés et que l'absence de perspectives d'évolution ainsi que la détérioration des conditions de travail incitent les plus jeunes à la mobilité.

\section{Les suppressions d'emplois : une déqualification largement ressentie par les ouvriers}

Les suppressions de postes intervenues depuis 2006, notamment depuis la fin 2008 ( $c f$. encadré 2) ont entraîné des bouleversements dans certaines usines pour le personnel ouvrier "survivant ", c'est-à-dire ceux qui restent après les réductions d'effectifs ; en effet, des activités ont disparu, provoquant des fermetures d'ateliers (de fonderie, de peinture, d'outillage...) et une partie de ce personnel a dû être reclassée, ce qui a eu un impact sur leur qualification.

Par ailleurs, dans toutes les usines étudiées, des réorganisations ont eu lieu en corollaire ; cette interdépendance entre ces réorganisations et les suppressions de postes a provoqué des changements dans le contenu du travail ouvrier.

depuis la fin 2005, en raison des délocalisations de production et aussi de la mévente de certains modèles de voitures, notamment ceux de haut de gamme. Alors que globalement en France, les années 2006 et 2007 sont considérées comme une période de reprise après la conjoncture morose des années 2004-2005 ( $c f$. Danzin, Simonnet, Trancart, 2011), ce sont des années noires pour l'emploi chez les équipementiers et les autres fournisseurs automobiles ; la récession qui a suivi la crise financière de 2008 a aggravé la situation et accéléré les suppressions d'effectifs. 


\section{Encadré 2}

\section{Les effets de la crise financière de 2008 sur l'emploi dans les usines étudiées}

Les personnes interrogées en 2010-2011 ont longuement parlé des effets de la crise financière de 2008 sur l'emploi. Ceux-ci ont été immédiats ou différés, de courte durée ou durables. Dès qu'elles ont été touchées par des baisses de commandes, toutes les usines ont renvoyé leurs intérimaires et leur personnel en CDD (contrat à durée déterminée), et recouru à du chômage partiel. La récession a provoqué une réduction de l'effectif en CDI (contrat à durée indéterminée) avec ou sans licenciements selon les usines.

Plusieurs usines constructeurs et fournisseurs ont proposé des départs ou des mutations dans le cadre d'un volontariat plus ou moins contraint, même celles qui ont été peu touchées par la récession. Celle-ci a pu représenter une occasion pour licencier, comme le suggèrent les propos d'un ouvrier et d'un médecin du travail dans cette usine fournisseur de 220 personnes peu affectée par la crise économique. Un licenciement de 81 personnes a eu lieu en 2009, «sous prétexte de la crise alors que la récession n'avait rien à voir avec ce licenciement » (un ouvrier). La décision d'effectuer un plan social avait été prise dès 2007, à la suite d'une étude qui montrait " qu'il fallait réduire les ratios et rentabiliser la boîte » (le médecin du travail). La reprise d'activité, provisoire ou durable, a entraîné une surcharge de travail, et un recours important aux heures supplémentaires. II n'y avait pas d'embauche pour remplacer les départs, ni de recours aux intérimaires si le surcroît de travail était jugé provisoire.

La mise en concurrence, au niveau mondial, des usines de la filière automobile a accéléré la mise en place de la lean production ( $c f$. encadrés 3 et 4), et les effets pervers de celle-ci dans un contexte de suppressions d'emplois ont été soulignés par nos différents interlocuteurs.

Leurs propos révèlent que ces réorganisations qui visent à réduire les effectifs ont entraîné une plus grande standardisation du travail, une intensification de celui-ci, une responsabilisation accrue du personnel ouvrier qui doit, dans certains cas, assumer de nouvelles tâches, notamment de gestion de la qualité ou de maintenance préventive, sans contreparties en termes de classification et de rémunération.

L'impact des suppressions d'emplois sur la qualification des ouvriers « survivants » est ainsi ambivalent. La standardisation du mode opératoire qui réduit l'autonomie de l'ouvrier entraîne une déqualification, largement ressentie. L'intensification du travail est aussi perçue comme un facteur de déqualification, la répétitivité des gestes étant accrue, d'où un moindre intérêt pour le travail effectué en raison du manque de temps et un sentiment de frustration, la priorité étant donnée à la productivité au détriment de la qualité. En revanche, l'ajout de tâches considérées comme très qualifiées, suite à des suppressions de postes en qualité ou dans l'encadrement, peut accroître les compétences de certains ouvriers ; mais si celles-ci ne sont pas reconnues en termes de classification et de rémunération, ces derniers ressentent une charge mentale accrue, une complexification de leur travail, qui est source d'angoisse et de stress, car ils ont une plus grande responsabilité avec une marge d'autonomie limitée. 


\section{Encadré 3}

\section{La lean production}

"Le modèle de la lean production s'apparente au modèle toyotien dont il constitue une adaptation dans le contexte de systèmes productifs nord-américain et européen » (Valeyre, 2010). II a été présenté au début des années 90 comme la seule voie à suivre en matière de performance industrielle dans l'ouvrage américain Le système qui va changer le monde, (Womack et alii, 1990), traduit en français en 1992. Le succès de cet ouvrage a suscité de nombreux débats, et des chercheurs ont montré qu'il existait d'autres modèles tout aussi performants dans l'industrie automobile (Boyer, Freyssenet, 2000).

Des travaux récents (Valeyre, 2010 ; Ughetto, 2012 ; Bourgeois, 2012 ; Morais, Aubineau, 2012), s'intéressent aux implications de ce modèle pour le travail. Le lean $\left.{ }^{*}\right)$ fait " de nouveau parler de lui depuis le milieu des années 2000 » (Ughetto, 2012, p. 149), puisqu'il est mis en place dans un nombre croissant d'entreprises, y compris dans le secteur tertiaire. Pour Fabrice Bourgeois (2012), ergonome, cette progression n'est pas seulement quantitative, mais aussi qualitative car les mutations générées sont "plus en profondeur ». "Le modèle Toyota s'est imposé comme la référence unique à atteindre » (p. 138), comme un moyen de "faire converger la rentabilité et l'amélioration des conditions de travail ».

Les liens entre la lean production, l'intensité du travail et les risques pour la santé des salariés, ont été mis en valeur par Antoine Valeyre qui s'appuie sur les résultats des enquêtes européennes sur les conditions de travail, notamment celle de 2005 (Valeyre, 2010). L'auteur distingue deux nouvelles formes d'organisation du travail, celle des organisations apprenantes où les salariés disposent d'une large autonomie (tâches non répétitives, peu de contraintes de rythme, niveau élevé d'initiative et de communication), et celle des organisations en lean production (autonomie beaucoup plus restreinte et contrôlée). Ces deux formes d'organisation sont comparées à deux formes plus anciennes, l'organisation taylorienne et les organisations en structure simple. Si les organisations apprenantes sont en rupture avec le modèle taylorien, les organisations en lean production en conservent de nombreux traits. Les conditions de travail sont particulièrement dégradées dans les organisations tayloriennes et dans celles en lean production. En 2005, les proportions de salariés européens déclarant que le travail porte atteinte à la santé étaient de $44 \%$ en lean production et de $43 \%$ en organisation taylorienne, contre un tiers pour l'ensemble des salariés.

(*) Dans les années 90 , le modèle qui s'appuyait sur le système de production de Toyota était appelé la "lean production », car il ne concernait que l'industrie. Actuellement, son champ d'application s'est élargi, et sont utilisés également les termes de lean manufacturing et de lean management. Pour simplifier, nous préférons comme de nombreux chercheurs et professionnels, qualifier ce modèle de lean, sans autre précision. 


\section{Encadré 4}

\section{L'exemple de la lean production dans l'automobile}

L'implication du lean sur le travail, mais de façon spécifique sur celui des ouvriers d'un constructeur automobile, est au cœur de l'analyse sur l'articulation entre l'ergonomie et le lean d'Alexandre Morais et de Raynald Aubuneau (2012). Ces deux ergonomes, salariés de ce constructeur, considèrent que c'est depuis 2005 que le lean est véritablement mis en œuvre dans le groupe, car les opérateurs doivent suivre strictement des modes opératoires formalisés. Le lean repose ici sur l'élimination de tout ce qui n'est pas valeur ajoutée, et le standard est l'expression de la meilleure pratique pour la rentabilité comme pour la qualité. La mission de l'ergonome chez ce constructeur est " d'œeuvrer pour que la performance des opérateurs soit améliorée en même temps que leur santé préservée tout au long de leur vie professionnelle » (p. 187). Or vers les années 2005 , les opérateurs ont commencé à exprimer des plaintes qui portaient surtout sur la charge mentale et organisationnelle. La fragilité principale dans l'application du lean est que sa mise en œuvre omet la philosophie du système qui est de faire remonter le moindre des problèmes pour le résoudre au plus tôt et de donner de l'importance au rôle de l'opérateur annoncé comme central. Celui-ci devrait participer à la transformation de son poste de travail, ce qui n'est pas le cas. Par ailleurs, le standard représente la meilleure pratique qui existe à un moment donné entre les opérateurs ; cela revient à ignorer les différences interindividuelles et intra individuelles. "Le standard est souvent défini au niveau du geste (augmentant ainsi le risque d'apparition de troubles musculo squelettiques...) et ne laisse pas de manceuvres, dont on sait pourtant qu'elles sont nécessaires pour faire face aux micro-aléas qui apparaissent au cours de l'activité » (p. 196). Tommaso Pardi (2009), qui a étudié l'usine Toyota en France, montre que les ouvriers travaillent dans un état d'urgence permanent, et que les consignes de sécurité sont de ce fait peu respectées; ce qui se traduit par des pathologies et un nombre d'accidents du travail étonnamment élevé compte tenu de la jeunesse de la main-d'œuvre. Le rejet du lean est très net également dans les entretiens que nous avons menés depuis 2006 avec des ouvriers, des médecins du travail, et des représentants syndicaux

\subsection{Une plus grande standardisation du travail ouvrier}

Les réorganisations de la production et du travail, depuis 2006, peuvent précéder les compressions d'effectifs, les accompagner, ou être réalisées à la suite de celles-ci. Les restructurations récentes accroissent les prescriptions, le mode opératoire devant être le même pour tous pour que le travail puisse être effectué par un intérimaire ou délocalisé. La mise en concurrence des usines au niveau mondial a entraîné un certain retour du taylorisme ; ce phénomène a déjà été mis en relief dans des travaux qui montraient que des expériences comme la mise en place d'îlots autonomes étaient abandonnées depuis le début des années $2000 \mathrm{au}$ profit d'une réinstallation du travail en ligne et d'une parcellisation des tâches, notamment dans les usines fournisseurs de garnissage de sièges (Gorgeu, Mathieu, 2008). Les propos d'ouvriers, de médecins du travail, et de syndicalistes, en 2010 et 2011, révèlent que la récession, en activant la diffusion du lean, a accéléré cette standardisation du travail. 
Le médecin d'un atelier protégé ${ }^{3}$, fournisseur qui livre en juste à temps une usine constructeur, s'inquiète de la mise en place du lean manufacturing qui vient d'être imposé par le constructeur " c'est-à-dire de la production maigre (lean production) dans un contexte de demande changeante, cela ne va pas améliorer les choses, bien au contraire. On pense à la place des autres pour chasser les mauvais gestes, on reconfigure en permanence, on fait la chasse au gaspillage. On mia montré un aménagement dans ce cadre. Je pense que cela enlève de l'autonomie aux gens. On leur demande de moins réfléchir et les chefs d'équipe auront une charge plus importante ".

La standardisation concerne les usines des constructeurs comme celles des fournisseurs, comme l'illustrent ces propos respectifs d'un médecin du travail et d'un ouvrier :

"Il y a une refonte des postes depuis 2009, et des spécialistes sont venus travailler sur la recomposition. Le discours officiel, c'est l'accroissement de la productivité, celle de la qualité, et l'amélioration des conditions de travail, mais le but c'est d'enlever des personnes. Il ne faut plus se déplacer et travailler tout le temps... on ne peut plus faire de l'avance. On met de l'ergonomie sur des postes de travail, des outils sont censés améliorer les conditions de travail mais c’est pour réduire le travail à son geste... c'est redevenu une chaîne à l'ancienne. On fait de la standardisation, c'est-à-dire que tous les ouvriers doivent avoir le même mode opératoire. Les gens travaillent tout le temps à la chaîne, et, sills veulent s'absenter, ils sont obligés de se faire remplacer. Les postes sont faits pour des gens standard. Il faut que le salarié soit standard." (Médecin du travail d'une usine de montage). Un ouvrier de 52 ans, salarié depuis 1978 d'une usine de montage appartenant à un autre constructeur, a été formé aux standards de travail lors de la récession pendant que d'autres étaient en chômage partiel. Pour lui, la standardisation qui se met en place depuis un an dans l'usine, "pour que tout le monde travaille pareil, selon les méthodes Toyota", vise à réduire les effectifs, "les standards, c'est pour économiser des hommes".

Cette standardisation est visible lors des visites de certaines usines de la filière automobile ; en effet, le " compactage ", c'est-à-dire la réduction des surfaces de production, élément essentiel du lean, entraîne un resserrement des postes de travail, une suppression des déplacements des opérateurs (jugés sans valeur ajoutée), puisque tout ce dont l'ouvrier a besoin se trouve sur son poste de travail. En montage, les lignes de production peuvent redevenir ainsi des chaînes à l'ancienne, avec pas ou peu de stocks intermédiaires, et l'impossibilité de s'absenter si on n'est pas remplacé. Les opérateurs doivent travailler au même rythme, faire tous les mêmes gestes; comme le précisait une ouvrière, "on est devenu des petits robots ». La visite dans une usine d'un centre de formation des intérimaires pour qu'ils acquièrent le «bon geste " pour tenir les cadences, a montré qu'il n'y a qu'une seule manière de visser ou de tenir le pistolet de peinture. Cette standardisation rend la polyvalence plus difficile car pour changer de poste, l'intérimaire doit connaître les bons gestes de chacun de ces postes.

3. Les ateliers protégés sont des unités de production qui dépendent du ministère du Travail. Ils recoivent des aides aux postes de travail et non aux personnes. Ce sont des usines dont le personnel est handicapé (handicaps divers), à l'exception du personnel d'encadrement. 
Les promoteurs du lean critiquaient le taylorisme et la spécialisation des tâches, en mettant en avant la polyvalence et la diversification des opérations ; or, une mise en place du lean avec comme seul objectif de réduire les effectifs réintroduit une forme de taylorisme démotivante et qui présente des risques pour la santé. Un ouvrage édité par Elsie Charron et Paul Stewart (2003) pointait déjà que ces pratiques détérioraient la qualité de la vie au travail dans le secteur automobile de plusieurs pays.

Une plus grande standardisation du travail ouvrier, c'est aussi ce que constatent Corinne Gaudart et Karine Chassaing (2011) qui ont étudié les modalités d'apprentissage des gestes à plusieurs années d'intervalle dans la même usine de montage d'un constructeur automobile français. Dans les années 90, ces ergonomes ont observé une action de formation " in situ ", c'est-à-dire sur les lignes de montage, visant à développer la polyvalence des ouvriers de production, puis plus récemment des formations intégrées à une « école de dextérité ", située à proximité des lignes de montage. Dans cette école, les opérateurs venaient s'entraîner aux " bons gestes ", dans un contexte de mise en place de nouveaux outils de gestion orientés vers la standardisation. L'absence de formalisation dans la première action de formation laissait des marges de manœuvre aux ouvriers alors que dans la seconde, la prescription ne laisse aucun choix à l'individu d'élaborer un mode opératoire différent. La mondialisation accroitt la standardisation du travail ouvrier, c'est une des conclusions des travaux d'Ariel Sévilla (2011), basés sur une observation participante dans deux usines de montage du même constructeur, l'une en France, l'autre au Brésil. La « dextérité » est une formation standard destinée à toutes les usines du groupe, quelle que soit leur localisation.

\section{Intensification du travail, mutations de postes et charges supplémentaires}

Même s'il n'y a pas de suppressions d'emplois en CDI, parce que les intérimaires servent de variable d'ajustement ${ }^{4}$, la diminution des effectifs utilisés se traduit par une intensification du travail, qui est très fortement ressentie par nos interlocuteurs, lors de nos entretiens de 2006-2007 et de 2010-2011. Le renvoi des intérimaires est une source d'inquiétude pour les ouvriers en CDI. Il annonce d'une part, une période d'incertitude et de mutations, de

4. Le nombre d'intérimaires utilisés dans les usines de la filière automobile a fortement progressé au cours des vingt dernières années. Au début, ce recours avait un double objectif : servir de variable d'ajustement en fonction du volume d'activité et permettre l'évaluation sur une durée longue pour sélectionner des ouvriers en vue d'une éventuelle embauche en CDI. Comme depuis une dizaine d'années, les embauches en CDI se sont raréfiées, les intérimaires n'ont plus la perspective d'être intégrés ; cependant, ils continuent à être très nombreux et à occuper des postes permanents, notamment les postes les plus pénibles et ceux qui risquent d'être délocalisés. Dans la décennie 2000, l'industrie automobile (les constructeurs et les équipementiers) est le secteur qui, selon le ministère du Travail, emploie le plus d'intérimaires, le taux de recours dépassant généralement $10 \%$ chaque trimestre (11,7\% au premier trimestre 2008). En revanche, en un an, de fin mars 2008 à fin mars 2009, quatre emplois intérimaires sur cinq ont été supprimés dans l'industrie automobile. 
détérioration de leurs conditions de travail car ils sont chargés d'assumer les opérations les plus pénibles réservées aux intérimaires, et d'autre part de déqualification puisque les postes tenus par les intérimaires sont considérés comme les moins qualifiés. Ainsi, cet ouvrier de 44 ans, interrogé en 2006, avait en charge le nettoyage des malaxeurs, qu'il n'avait pas avant : "Vue mon ancienneté, c'était une petite faveur, car cela était fait par des intérimaires. Il n'y a plus d'intérimaires depuis le rachat par la nouvelle société, alors qu'ily en avait beaucoup avant (140 en 2004), Adecco ayant un implant dans l'usine. Ce sont maintenant les anciens qui font le travail des intérimaires. " Fin 2010, une ouvrière de 38 ans ayant plus de dix ans d'ancienneté dans son usine d'équipement automobile précise qu'elle a particulièrement ressenti à la fois une intensification du travail et un déclassement, lorsqu'elle a été remise en production pour faire un travail d'intérimaire fin 2008 début 2009 : "C'est un moment dur où les intérimaires ont été renvoyés. Ils ont comblé les trous. Je faisais leur boulot. Je remplaçais en production quatre intérimaires. "

Un article récent (Dubreuil, Gautier, 2011) sur une usine de production automobile montre que le renvoi des intérimaires, en 2008, a eu des effets sur la santé des ouvriers et a provoqué une insatisfaction due à la fois à la détérioration de leurs conditions de travail et à un sentiment de déqualification. "Les difficultés de l'usine seraient liées à un arrêt trop brutal du recours aux intérimaires. Leur disparition révèle l'impact de certains postes de travail sur la santé des ouvriers" (p. 64). Les auteurs ajoutent que "réaffecter des salariés 'embauchés'sur des postes durs devenus des postes d'intérimaires est majoritairement perçu comme dégradant».

Les entretiens effectués en 2010-2011 mettent en relief les liens entre suppressions d'emplois et intensification du travail. Ils montrent que la détérioration de l'emploi entraîne des mutations de postes qui sont généralement mal vécues par le personnel concerné, surtout lorsqu'elles se traduisent par une déqualification ; par exemple, quand des professionnels de maintenance ou des animateurs d'équipes se retrouvent en production. Les suppressions de postes peuvent aussi accroître les compétences du personnel ouvrier sans contreparties en termes de qualification et de salaire, car des tâches supplémentaires leur sont imposées qui intensifient leur travail et augmentent leurs responsabilités. C'est le cas dans cette usine fournisseur où, depuis la réduction des effectifs (51 départs sur un effectif de 107 personnes avant la récession), les ouvriers de production doivent s'occuper du conditionnement et de la gestion de la qualité, la fonction qualité qui comptait un effectif de trois personnes n'existant plus. Il leur faut assumer en plus de leur travail de production la vérification des cotes avec des instruments, le contrôle visuel, la mise à jour des documents, et, en cas de problèmes qualité, la remontée des informations au chef d'équipe et, au-dessus, au chef de poste. 


\section{Un déclassement et une insatisfaction 3 qui incitent à la mobilité}

La majorité des ouvriers et des ouvrières interrogés depuis 2006 manifestent une forte insatisfaction en raison à la fois de la détérioration de leurs conditions de travail et de la faible reconnaissance de leurs compétences, celles-ci étant évaluées chaque année par leur supérieur hiérarchique. Les changements qu’ils ont connus depuis plusieurs années (de groupe, de management, d'organisation, etc.), et les continuelles réductions de personnel rendent leur travail plus pénible, tendent à remettre en cause leur qualification et ne leur laissent entrevoir aucune perspective d'évolution satisfaisante. Dix-huit des dix-neuf ouvriers et ouvrières interrogés fin 2010 début 2011 avaient ainsi répondu lors de l'enquête SUMER que leurs perspectives d'évolution étaient « faibles » ou « insuffisantes » en regard des « efforts consentis ». Lors de l'entretien, plusieurs personnes reconnaissent avoir refusé des propositions par crainte d'une charge de travail accrue, de responsabilités trop lourdes, de conflits latents, d'une obligation de réserve, et de contreparties financières insuffisantes.

Si des personnes ont refusé des changements considérés comme des promotions mais qui leur paraissaient leur apporter plus d'inconvénients que d'avantages, d'autres ont été déclassées. Les exemples de déclassements analysés ci-dessous sont en lien avec la récession, mais déjà en 2006-2007 des cas de déclassements, suite à des suppressions de postes, avaient été évoqués, notamment ceux de peintres qui se sont retrouvés opérateurs de production, avec un coefficient de qualification et un salaire moindres, "tu prends ou tu dégages», selon l'expression d'un ouvrier d'une usine fournisseur. La fermeture d'un atelier fonderie dans une usine constructeur en 2005, qui a provoqué une forte diminution de l'effectif sans licenciements économiques, a été aussi un choc psychologique traumatisant pour le personnel reclassé dans d'autres ateliers de l'établissement. Les fondeurs ont perdu leur autonomie et ont été mis sur les postes les moins complexes, ce qui est ressenti comme une déqualification, comme l'illustrent les propos de cet ouvrier de 44 ans, qui regrette à la fois l'ambiance familiale de la fonderie et la responsabilité qu’il avait " de gérer son sable ». "On avait du goût à venir travailler malgré que c'était pénible... vous savez. Maintenant, question intégration, c'est négatif.. même si vous avez vingt-quatre ans d'ancienneté... et bien je me considère comme intérimaire parce que je débarque et puis je suis obligé de recommencer tout, de refaire mes preuves ». Ce fondeur se retrouve sur une ligne de fabrication à un poste de contrôle de pièces.

\subsection{Des exemples de déclassement}

Cinq personnes 5 , trois ouvrières et deux ouvriers, parmi les dix-neuf interrogées fin 2010 début 2011, ont fait état de déclassement. Ce ressenti peut s'expliquer principalement par l'inadéquation entre le niveau du diplôme et l'emploi occupé, ce qui est le cas de Jean, ou

5. Les prénoms des personnes ont été changés. 
le manque de reconnaissance du diplôme et de la qualification exercée, ce qui est le cas de Sophie, ces deux jeunes n'ayant par ailleurs aucune perspective d'évolution dans l'entreprise où ils travaillent. Les trois personnes plus âgées, Marie, Jeanne et Henri, ont connu un changement de poste considéré comme un déclassement, car il remettait en cause leur expérience et leur qualification.

" Je commence à en avoir marre, je suis déclassée " (Sophie, 28 ans, titulaire d'un DUT - diplôme universitaire de technologie - de maintenance)

Cette jeune femme, qui travaille depuis 2008 chez un fournisseur de pièces en matières plastiques pour l'automobile, exerce le métier de régleur en injection. Elle monte et démonte les moules, elle fait du réglage et s'occupe des machines de deux ateliers (injection et assemblage) ; elle surveille et intervient sur les machines pour éviter que le process ne dérive car les problèmes liés à la qualité sont fréquents dans cette activité. Son chef d'atelier étant parti en retraite (depuis deux ans) et n'étant pas remplacé, elle occupe de fait cette fonction sans en avoir ni le titre ni le salaire. Le directeur de l'entreprise, lors de son embauche, lui avait signifié qu'elle deviendrait chef d'atelier : "Je ne suis pas au poste que je devrais être car je suis trop diplômée, j’ai fait un DUT en maintenance et j’ai un bac en plasturgie... Je commence à en avoir marre, je me sens déclassée car aujourd'hui le métier de régleur s'apprend sur le tas.. J'ai été trompée... Je ne pense pas rester dans cette entreprise." Par ailleurs, elle a de mauvaises conditions de travail : "Je respire des vapeurs d'huile et il n'y a pas de hotte aspirante et il y a beaucoup de poussière, il y a aussi des vibrations, c'est très bruyant... Je me rends compte qu'au niveau de la santé rien n'est bon là-bas, surtout ces derniers temps... depuis un an. " La situation a empiré suite à des plans sociaux qui ont abouti au départ de 45 personnes en 2009, et à une intensification du travail, le personnel restant étant contraint à faire des heures supplémentaires : "Si on refuse, il y a allusion au licenciement par le directeur... il y a un ras le bol général... l'ambiance n'est pas terrible... il $y$ a trois régleurs, sil en manque un c'est aux deux autres de faire le travail. " Depuis un an, elle avait une sciatique par intermittence, et tout récemment mal aux mains à force de serrer et de desserrer les moules : «ça me fait peur. » Elle commence à prendre conscience qu'elle ne fera pas un métier en rapport avec ses diplômes, et que ses ennuis de santé sont liés à ses mauvaises conditions de travail. Au moment de l'entretien, fin 2010, elle venait de créer son auto-entreprise qui fonctionne toutes les fins de semaine; elle donne des cours de cuisine et met des recettes sur internet : "Je pense que je serai plus épanouie. Si ça fonctionne, je quitterai l'entreprise, sinon, si ça marche pas, je chercherai un travail en journée selon mes compétences. »

\section{Un emploi de cariste, un gagne-pain " déclassant " pour Jean, 32 ans, titulaire d'un BTS (brevet de technicien supérieur)}

La formation initiale de Jean, titulaire d'un BTS de tournage formage, et son expérience d'enseignant vacataire ne le prédisposaient pas à l'emploi de cariste chez un fournisseur automobile, qu'il exerce depuis trois ans et qu'il essaie de quitter. Son cas est très différent de celui de Sophie. Celle-ci est déclassée parce que son entreprise ne veut pas reconnaître, 
en termes de qualification et de rémunération, ses compétences de chef d'atelier, métier qu'elle exerce et qui est en correspondance avec ses diplômes, alors que Jean a un emploi de cariste sans lien avec sa formation, son diplôme et son expérience. Cet emploi lui permet de faire vivre sa famille (il a deux jeunes enfants), et il l'occupe faute de mieux, son projet de devenir professeur n’ayant pu se réaliser en raison de la réduction du nombre d'enseignants. Depuis plusieurs mois, il cherche un emploi de technicien des méthodes mais les annonces sont très rares dans la région et dix ans d'expérience sont exigés.

Son emploi de cariste dans la filière automobile lui a occasionné beaucoup d'ennuis de santé et il a eu un accident de travail avec 45 jours d'arrêt pour dorsalgie. C'est également pour cette raison qu'il souhaitait changer d'activité. Au moment de l'entretien, fin 2010, il envisageait de négocier son départ et de faire une formation avec l'espoir de trouver un emploi en lien avec son diplôme.

\section{Le cas de deux ouvrières animatrices redevenues opératrices de production fin 2008}

Ces deux femmes, Marie (38 ans) et Jeanne (49 ans) travaillent dans la même usine fournisseur où elles possèdent une forte ancienneté. Elles étaient animatrices, c'est-àdire responsables d'une équipe d'opérateurs sans avoir de rôle hiérarchique, depuis plusieurs années, mais à la fin 2008, elles sont " redescendues » opératrices de production sans perte de salaire. La raison officielle de ce changement était un trop grand nombre d'animateurs par rapport à un effectif en diminution à cause de la crise. Selon ces deux ouvrières, leur comportement insoumis et les mauvaises relations qu'elles entretenaient avec leur chef d'équipe expliquent leur déclassement. Celui-ci n’a été que provisoire pour la plus jeune qui est redevenue animatrice après avoir changé de chef d'équipe. En revanche, Jeanne sait qu'elle ne sera plus jamais promue à cette fonction en raison de son âge : "Les gap leaders de nos âges sont remplacés par d'autres plus jeunes."

Ces deux ouvrières ont des ennuis de santé en raison notamment de l'accélération des cadences et de la diminution des effectifs. Jeanne a une tendinite et Marie une entorse cervicale et des troubles du sommeil. Cette dernière envisage de partir car elle se sent très fatiguée " dès le mercredi " et sa vie familiale en pâtit. Elle aspire à travailler la journée et non plus en postes de 2/8 (une semaine le matin, une semaine l'après-midi) et à pouvoir ainsi se consacrer à ses jeunes enfants.

Redevenir cariste, après avoir eu la responsabilité d'une équipe, une "dégradation » pour Henri, 52 ans, au chômage au moment de l'entretien fin 2010

La vie professionnelle de cet ouvrier a basculé en mai 2009, après ses trois quart d'heures d'entretien annuel avec son supérieur hiérarchique. Ce responsable de la logistique, "bras droit du nouveau directeur qui voulait révolutionner la logistique ", lui a annoncé qu'il allait, dans le cadre de ce bouleversement, redevenir cariste, c'està-dire être dégradé. Henri avait en effet quitté cet emploi pour évoluer, et devenir depuis sept ans responsable d'une équipe de logistique liée à un constructeur automo- 
bile allemand ${ }^{6}$. "Je reculais de trente ans en arrière... à 52 ans, il n'en est pas question." Ses conditions de travail se seraient détériorées car il aurait eu à travailler dans un hangar ouvert à tous les vents (chaud l'été, froid l'hiver). Cette annonce a été un choc car il ne s'attendait pas à cette proposition. Voyant qu'il n'y avait aucune possibilité de négociation (aucune formation à des logiciels ne lui a été proposée, notamment Excel), il a demandé à changer de secteur à la directrice des ressources humaines. Elle lui a proposé un poste sur des machines automatiques en 3/8 (une semaine le matin, une semaine l'après-midi, une semaine la nuit), un emploi de "presse bouton ", considéré comme moins qualifié que celui qu'il avait : "J'ai été dégradé de P3 à P2. "Il s'est senti " rabaissé » et sans avenir car il n'avait aucune perspective de progression : "Avec mon caractère, je ne pouvais pas durer six mois de plus. "Cette situation s'est prolongée de mai à septembre 2009. Comme il était très atteint psychologiquement, le médecin du travail l'a mis en inaptitude, et l'entreprise l'a licencié en mars 2010. Depuis cette date, il est au chômage, inscrit à Pôle emploi, et reconnu travailleur handicapé depuis novembre 2010.

\subsection{Quitter la filière automobile : un souhait exprimé surtout par les plus jeunes}

Les entretiens réalisés depuis 2006 montrent que les personnes les plus jeunes rejettent, pour la plupart, à la fois le travail ouvrier et les modes de management des usines de la filière automobile, ce qui les conduit à envisager de quitter le plus rapidement possible cette filière, notamment depuis la crise structurelle que celle-ci connait. En 2006-2007, période où nous avions pu réaliser des entretiens avec des intérimaires, les quatre intérimaires les plus jeunes qui avaient obtenu des missions à la fois dans des usines constructeurs et dans des usines fournisseurs, étaient unanimes pour rejeter cette filière ; en effet, ils ne voulaient plus travailler chez les équipementiers et savaient qu'ils ne seraient jamais embauchés en CDI chez les constructeurs. Un cariste avait interrompu une mission chez un équipementier pour des raisons à la fois de sécurité et d'intensité du travail. Il préférait être au chômage que de prendre le risque de provoquer un accident du travail ou d'en être la victime, et il voulait devenir ambulancier. Une opératrice réalisant des opérations d'assemblage chez un fournisseur continuait sa mission, mais se renseignait sur une formation de télé-opératrice; elle disait qu'elle n'accepterait plus de missions dans cette filière car elle avait, depuis quelques mois, un début de tendinite, suite à ses gestes répétitifs sous fortes contraintes de temps. Les deux autres avaient déjà quitté la filière en grande partie en raison de leurs conditions de travail chez les fournisseurs où ils avaient effectué leurs dernières missions, et ils suivaient une formation dans le bâtiment. En 2010-2011, trois personnes en CDI de moins de 40 ans, Sophie, Jean et Marie, avaient des projets de

6. Sa fonction consistait à réceptionner les produits finis de l'entreprise et à les envoyer à temps chez le client pour ne pas arrêter la ligne de montage des véhicules. Il avait un bureau, et n'était qu'épisodiquement sur le terrain où trois caristes travaillaient sous sa responsabilité. 
mobilité plus ou moins formalisés parce qu'elles avaient connu un déclassement et rencontraient des ennuis de santé liés à leur travail.

\section{Conclusion}

Le vécu des salariés victimes de restructurations qui se retrouvent au chômage a donné lieu à plusieurs recherches depuis le début des années 2000 ( $c f$. notamment Linhart, Rist, Durand, 2002). Le cas des salariés qui restent dans l'entreprise après d'importantes suppressions d'emplois a été peu étudié. Marie Raveyre (2005) analyse l'impact d'une restructuration hospitalière sur les conditions de travail. Elle montre que la charge de travail s'accroît pour le personnel restant, entraînant une exacerbation des tensions, et une insatisfaction généralisée. Les travaux réalisés depuis 2006 sur les conditions de travail des ouvriers de la filière automobile aboutissent à des résultats comparables. La concomitance entre les suppressions d'emplois et la diffusion des méthodes Toyota détériore les conditions de travail dans les usines, en rognant sur les espaces de liberté des ouvriers. Christian Corouge, ouvrier dans une usine de montage constructeur depuis quarante ans, dresse le même constat, quand Michel Pialoux, en novembre 2010, évoquant les changements dans l'usine, lui demande sur quoi il mettrait l'accent (Corouge, Pialoux, 2011) : "Sur la disparition de tous les espaces de liberté quion pouvait avoir... t'avais une marge de manouvre très différente. Alors que la y'a aucune échappatoire, y a plus de possibilités de négociation avec la maîtrise. Nous, on n'avait jamais connu ça, et c’est très pesant à vivre. " (p. 443). Les restructurations provoquent des troubles et des pathologies en lien avec le travail, dus à la fois aux exigences accrues et à une marge d'initiative réduite, ce cumul étant en effet source de tension et de pathologies, comme le montre le modèle de Karasek ${ }^{7}$. Dans le cas des ouvriers, les risques psychosociaux s'ajoutent à des pénibilités physiques et organisationnelles, avec notamment le travail posté ou de nuit (Waltisperger, 2007). La détérioration des conditions de travail des ouvriers renforce ainsi les inégalités sociales très prononcées selon les catégories professionnelles en matière de mortalité prématurée (entre 45 ans et 59 ans) et d'usure de l'organisme ${ }^{8}$.

Les travaux qualitatifs sur la filière automobile, menés depuis 2006, mettent en relief une relation étroite entre la dégradation de l'emploi et la détérioration des conditions de travail et montrent que les suppressions de postes ont un impact négatif sur la qualification

7. Karasek est un psychosociologue nord-américain qui montre, à partir de travaux statistiques, que le «job strain " (qui peut être traduit en français par "tension au travail») résulte de la combinaison de fortes exigences et de faibles marges de manœuvre, cette tension étant accrue lorsque le salarié ne bénéficie pas de soutien social de la part de ses supérieurs hiérarchiques et de ses collègues. On peut alors parler de risques psychosociaux qui peuvent entraîner des pathologies spécifiques, essentiellement des troubles musculo-squelettiques (TMS), cardio-vasculaires et psychiques.

8. La différence d'espérance de vie à 35 ans entre cadres et ouvriers est en France de sept ans et, d'après l'Institut national d'études démographiques (INED), après 50 ans, les ouvriers passent en moyenne plus de la moitié de leur vie en mauvaise santé, contre un tiers de leur vie pour les cadres (Cambois, Barnay, Robine, 2010). 
ouvrière. En France, la désindustrialisation et les suppressions d'emplois qu'elle provoque auraient ainsi des effets néfastes à la fois pour le personnel victime de celles-ci et pour celui qui reste dans les usines après les restructurations. Elles entraineraient un déni de la formation initiale et continue et de l'expérience professionnelle qui démobiliserait le personnel et dont les effets à terme seraient préjudiciables et mériteraient d'être plus abondamment explorés. Un des moyens de sauvegarder l'industrie, comme semble l'indiquer l'exemple de l'Allemagne, serait d'éviter de supprimer des emplois, en privilégiant le chômage partiel et en reconnaissant les qualifications ouvrières.

\section{Bibliographie}

Ardenti R., Mathieu R. Gorgeu A. (2010), "Caractère soutenable du travail et trajectoires ouvrières : Etudes de cas dans la filière automobile et les industries agroalimentaires ", Sociétés contemporaines, $\mathrm{n}^{\circ} 78 \mathrm{pp} .87-113$.

Bourgeois F. (2012), "Que fait l'ergonomie que le lean ne sait/ne veut pas voir ?", Activités, Vol. 9 n $^{\circ}$ 2, pp. 138-147.

Bouvier G., Pilarski C. (2008), « Soixante ans d'économie française ; des mutations industrielles profondes ", INSEE Première, $\mathrm{n}^{\circ} 1201$, juillet, $4 \mathrm{p}$.

Boyer G., Freyssenet M. (2000), Les modèles productifs, Paris, La Découverte, 128 p.

Cambois E., Barnay T., Robine J.-M. (2010), "Espérance de vie, espérances de vie en santé et âges de départ à la retraite : des inégalités selon la profession en France ", Retraite et Société, n 59, pp. 194-205.

Charron E., Stewart P. (2003), Work and Employment:Relations in the Automobile Industry, Palgrave MacMillan, 297 p.

Corouge C., Pialoux M. (2011), Résister à la chaîne, Agone, " Mémoires sociales », 464 p.

Danzin E., Simonnet V., Trancart D. (2011), "Quels effets de la crise sur les trajectoires professionnelles des jeunes? " Connaissance de l'Emploi $\mathrm{n}^{\circ} 82,4 \mathrm{p}$.

Demmou L. (2010), «La désindustrialisation en France », Documents de Travail de la DG Trésor, $\mathrm{n}^{\circ}$ 2010/01, juin, $52 \mathrm{p}$.

Dubreuil F., Gautier B. (2011), "Mesure de la santé et maladie de la mesure : le contrôle des corps dans une usine de production automobile ", Sociologies Pratiques, $\mathrm{n}^{\circ} 22$, pp. 63-78.

Duval G. (2010) «2010, priorité à la lutte contre le chômage ", Alternatives économiques $\mathrm{n}^{\circ} 287$, janvier.

Gaudart C., Chassaing K. (2011), “Formation 'in situ' et 'école de dextérité' dans l'automobile : analyse des modalités d'apprentissage et de leurs coûts pour les opérateurs ", 
in Molinié A.-F., Gaudart C., Pueyo V. (coord.), La vie professionnelle, âge, expérience et santé à l'épreuve des conditions de travail, Toulouse, Octarès.

Gauron A. (2010), "Désindustrialisation et choix politiques ", Problèmes économiques, $\mathrm{n}^{\circ} 3003,29$ septembre (repris d'un article «Croire en l'avenir de l'industrie », Societal, $\mathrm{n}^{\circ} 69$, troisième trimestre 2010).

Gorgeu A., Mathieu R. (2008), "La déqualification ouvrière en question ", Formation Emploi, n 103, pp. 83-100.

Greenam N., Kalugina E., Walkowiak E. (2011), « La dégradation de la qualité de vie au travail en Europe entre 1995 et 2005 », Connaissance de l'emploi, n 84, 4 p.

Linhart D., Rist B., Durand E. (2002), Perte d'emploi, perte de soi, Paris, Eres, "Sociologie clinique ", $214 \mathrm{p}$.

Mathieu R., Gorgeu A. (2012), "Les effets de la récession sur le travail ouvrier dans la filière automobile en France : regards croisés d'ouvriers, de représentants syndicaux et de médecins du travail ", Les mondes du travail, n 12, pp. 19-31.

Morais A., Aubineau R. (2012), "Articulation entre l'ergonomie et le lean manufacturing chez PSA ", Activités, Vol. 9, n², pp. 179-197.

Pardi T. (2009), "Travailler chez Toyota : de l'emploi à vie à la course à la survie ", Revue de l'IRES, n 62, pp. 39-70.

Raveyre M. (2005), «Le travail dans le management des restructurations : entre déni et omission ", La Revue de l'IRES, n 47, pp. 95-115.

Sevilla A. (2011), " Former pour gérer la main-d'œuvre dans l'industrie automobile au Brésil et en France ", Connaissance de l'emploi, n 79, 4 p.

Ughetto P. (2012), «Le lean : pensée et impensé d'une activité sans relâchement », Activités, Vol. 9, n 2, pp. 148-167.

Valeyre A. (2010), Nouvelles formes d'organisation du travail et santé des salariés en Europe, communication pour le colloque du GRIS, «Organisation, gestion productive et santé au travail ", université de Rouen, 16 et 17 décembre (à paraître).

Waltisperger D. (2007), "Les ouvriers sont stressés eux aussi », Santé et Travail, n 57.

Womack J.P., Jones D.T., Roos D. (1990), Le système qui va changer le monde, Trad. française, Paris, Dunod, 1993, 349 p. 
Revue trimestrielle bilingue publiée depuis 1945 par le Département des relations industrielles de I'Université Laval

NUMÉRO THÉMATIQUE

Le Réseau de recherche en santé et sécurité du travail du Québec : un bilan de dix ans

\section{The Quebec Occupational Health and Safety} Research Network: A Ten Year Update

LISE DESMARAIS ET GABRIELLE LEGENDRE

ARTICLES

Le transfert de connaissances au RRSSTQ : bilan et perspectives

M. LORTIE, L. DESMARAIS, C. FAYE, É. LAROCHE ET I. FAURIE

Les conditions de travail au Québec : une analyse différenciée selon les groupes d'âge

É. LEDOUX, P.-S. FOURNIER, D. CHAMPOUX, P. PRUD'HOMME, M. LABERGE, C. AUROUSSEAU, S. OUELLET ET C. CHATIGNY

From Serious Shortage of Researchers to OHS Law Changes and Growing Industrial Needs: A Dynamic and Quiet Force

S. NADEAU, J. FATISSON, A. BADRI AND B. EMAMI-MEHRGANI

Advancement in Quebec Research on the Prevention of Risks Related to Occupational Exposure to Nanomaterials P. DOLEZ, Y. CLOUTIER, A. NOËL, L. TABET, D. GAUTRIN, M. DEBIA, G. L'ESPÉRANCE, S. HALLÉ, A. BAHLOUL AND L. VINCHES

Quebec Research on Work-related Musculoskeletal Disorders:

Deeper Understanding for Better Prevention

J. N. CÔTÉ, S. NGOMO, S. STOCK, K. MESSING, N. VÉZINA, D. ANTLE, A. DELISLE, M. BELLEMARE, M. LABERGE AND M. ST-VINCENT

Santé organisationnelle : où en sommes-nous et vers où allons-nous au Québec? V. DAGENAIS-DESMARAIS, M.-È. DUFOUR, F. ST-HILAIRE ET R. HÉBERT

Bilan relatif aux dispositions de la LSST : vers une application intégrale? G. BARIL-GINGRAS, M. VÉZINA ET K. LIPPEL

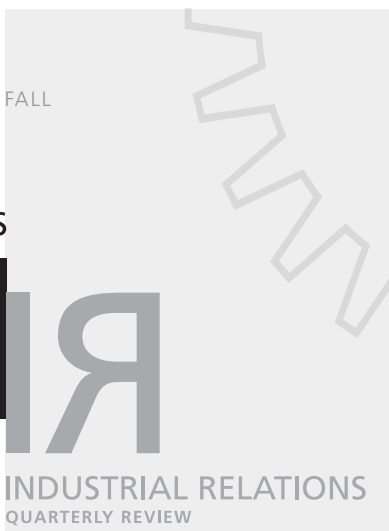

A bilingual quarterly published since 1945 by the Industrial Relations Department, Université Laval

\section{RI/IR EN LIGNE}

$\mathrm{RI} / \mathrm{R}$ est disponible en ligne sur le site Erudit : www.erudit.org/revue/ri

Pour abonnement à la version numérique, contacter Érudit.

Pour consulter les sommaires des numéros publiés, les résumés d'articles ou pour vous abonner à la version papier, visitez notre site Internet :

www.riir.ulaval.ca

\section{RI/IR ONLINE}

$\mathrm{RI} / \mathrm{IR}$ is available on line on Erudit website at: www.erudit.org/revue/ri

To subscribe to the online version, please contact Erudit.

Visit our website for contents listings, abstracts, or to subscribe to the print edition:

www.riir.ulaval.ca

\section{RELATIONS INDUSTRIELLES} INDUSTRIAL RELATIONS

Pavillon J.-A.-DeSève 1025, avenue des Sciences-Humaines Bureau 3129, Université Laval Québec (Québec) Canada G1V 0A6 TÉLÉPHONE : (418) 656-2468 COURRIEL / E-MAIL : relat.ind@rlt.ulaval.ca

www.riir.ulaval.ca 